\title{
Meta-analysis of trial data may support a causal role of hyperglycaemia in cancer. Reply to Yang XL, Ma RCW, Chan JCN [letter]
}

\author{
J. A. Johnson • S. L. Bowker
}

Received: 17 November 2010 / Accepted: 19 November 2010/Published online: 25 December 2010

(C) Springer-Verlag 2010

Keywords Cancer $\cdot$ Hyperglycaemia $\cdot$ Meta-analysis . Randomised controlled trials · Type 2 diabetes

To the Editor: We thank Yang and colleagues for their interest in our recent publication [1]. It is interesting how different investigators can view the same data and arrive at such different conclusions, influenced perhaps by their underlying beliefs. Yang and colleagues appear to believe that hyperglycaemia is causally linked to cancer risk [2], a belief that is in opposition to the evidence presented in our meta-analysis of large randomised trials of glucoselowering strategies for patients with type 2 diabetes. They present four arguments in support of their belief.

They first indicate that our meta-analysis lacked sufficient power to reach a conclusion of no difference in cancer risk between patients under intensified glycaemic control compared with conventional treatment. They indicate that the comparison 'had zero power at a type I error rate of 5\% to make a conclusion of equivalence'. In fact, we didn't make a conclusion of equivalence. We presume they meant to say the meta-analysis had zero power to detect such a difference given a type 1 error rate of 5\%. And the difference to which they are referring is the cancer incidence rates of 8.44 per 1,000 person-years in the control arms and 7.44 per 1,000 person-years in the intensified treatment arms. We agree that our metaanalysis is underpowered to detect this difference as being statistically significant. There is, of course, little value in

J. A. Johnson $(\bowtie) \cdot$ S. L. Bowker

Department of Public Health Sciences, School of Public Health, 2-040 Health Research Innovation Facility, University of Alberta, Edmonton, AB, Canada T6G 2E1

e-mail: jeff.johnson@ualberta.ca post hoc power calculations [3, 4], but the real question is, why pursue the statistical significance of such a small difference at all? A major element of a power calculation is the effect size of interest; in this case, the absolute difference is $0.1 \%$, which translates to a number-neededto-treat (NNT) of 1,000. By all standards this would be considered a trivial difference. In fact, given this trivial difference, a study that would conclude such a difference was statistically significant at conventional levels, with $80 \%$ power, would require 44,700 subjects in each group, followed for 5 years [5]. Pursuing nearly half a million person-years of follow-up hardly seems feasible or worthwhile.

Yang et al. also suggest that the cancer incidence was likely to have been underestimated, given the study designs limiting follow-up to other clinical events. It appears, however, that these rates are similar to those reported in observational studies, such as the Hong Kong Diabetes Registry, where cancer incidence amongst a cohort of type 2 diabetes patients was reported to be 5.8-9.7 per 1,000 person-years [6]. Furthermore, while there may be a potential for limited follow-up, this would be a nondifferential bias, since it would be true for both the control and intensified treatment arms; thus the interpretation of the OR is not affected. Furthermore, it is not an issue for the outcome of cancer mortality in the other meta-analysis we presented [1].

For their third argument, Yang et al. suggest that the results of the meta-analysis are consistent with epidemiological evidence of an association. We do not disagree that there is a strong and consistent epidemiological association between glycaemia and cancer risk. As we indicated, however [1], the well-known concern of potential confounding in epidemiological studies was the reason for 
conducting the meta-analysis of controlled experimental data. With that, it does not appear that active reduction of elevated blood sugar has an important causal relationship with cancer risk. This is emphasised by the above point, that, while the non-significant OR of 0.91 leaves open the possibility of some risk difference, the magnitude of the absolute difference is trivial. Yang and colleagues go on to state that 'if the causal role of glycaemic control in cancer is proven, there are even more reasons to advocate for early attainment of glycaemic control' [2]. This is, of course, a major speculation and reminiscent of the call for intensified glycaemic control to reduce the risk of macrovascular complications. The data from these major trials indicate that while there are similarly strong and consistent epidemiological associations, cancer risk, like cardiovascular risk, is not reduced when glucose control is actually improved.

Finally, Yang and colleagues argue for a biological link between hyperglycaemia and cancer risk, referring to a series of epidemiological studies of linking triacylglycerol, LDL- and HDL-cholesterol, and albuminuria to cancer risk in patients with type 2 diabetes. These epidemiological studies would suggest potential for many different biological processes, however, and not just hyperglycaemia. Regardless, such epidemiological associations would benefit from further confirmation with higher levels of evidence to draw inferences related to causality.

While the relationship between type 2 diabetes and cancer is consistent and reasonably strong, the underlying mechanisms are still not clear, and at this early stage of investigation it is important to consider all possibilities. Cancers are heterogeneous, and there may be specific tumours for which cell growth and differentiation is facilitated by hyperglycaemia. However, the accumulation of experimental and epidemiological evidence is more consistent with the hyperinsulinaemia hypothesis, and less so with the hyperglycaemia hypothesis [7]. The evidence from randomised controlled trials summarised in our metaanalyses would further support this.

Duality of interest The authors declare that there is no duality of interest associated with this letter.

\section{References}

1. Johnson JA, Bowker SL (2010) Intensive glycaemic control and cancer risk in type 2 diabetes: a meta-analysis of major trials. Diabetologia. doi:10.1007/s00125-010-1993-3

2. Yang XL, Ma RCW, Chan JCN (2011) Meta-analysis of trial data may support a causal role of hyperglycaemia in cancer. Diabetologia. doi:10.1007/s00125-010-2017-0

3. Levine M, Ensom MHH (2001) Post hoc power analysis: an idea whose time has passed. Pharmacotherapy 21:405-409

4. Goodman SN, Berlin JA (1994) The use of predicted confidence intervals when planning experiments and the misuse of power when interpreting the results. Ann Intern Med 121:200-206

5. Schoenfeld DA, Richter JR (1982) Nomograms for calculating the number of patients needed for a clinical trial with survival as an endpoint. Biometrics 38:163-170

6. Yang X, Ko GT, So WY et al (2010) Associations of hyperglycaemia and insulin usage with the risk of cancer in type 2 diabetes: the Hong Kong Diabetes Registry. Diabetes 59:12541260

7. Johnson JA, Pollak M (2010) Insulin, glucose and the increased risk of cancer in patients with type 2 diabetes. Diabetologia 53:2086-2088 